\title{
Sexual Robots: the Social-Relational Approach and the Concept of Subjective Reference
}

\author{
Piercosma Bisconti Lucidi ${ }^{1}$ and Susanna Piermattei ${ }^{2}$ \\ ${ }^{1}$ Sant'Anna School of Advanced Studies, DIRPOLIS Institute, Pisa, Italy \\ piercosma.biscontilucidi@santannapisa.it \\ ${ }^{2}$ Sapienza University of Rome, Department of Psychology, Rome, Italy \\ susannapiermattei@gmail.com
}

\begin{abstract}
In this paper we propose the notion of "subjective reference" as a conceptual tool that explains how and why human-robot sexual interactions could reframe users approach to human-human sexual interactions. First, we introduce the current debate about Sexual Robotics, situated in the wider discussion about Social Robots, stating the urgency of a regulative framework. We underline the importance of a social-relational approach, mostly concerned about Social Robots impact in human social structures. Then, we point out the absence of a precise framework conceptualizing why Social Robots, and Sexual Robots in particular, may modify users' sociality and relationality. Within a psychological framework, we propose to consider Sexual Robots as "subjective references", namely objects symbolically referring to human subjects: we claim that, for the user experience, every action performed upon a Sexual Robot is symbolically directed toward a human subject, including degrading and violent practices. This shifting mechanism may transfer the user relational setting from human-robot interactions to human-human interactions.
\end{abstract}

Keywords: Social Robots, Ethics of Technology, Psychology of HCI, HumanRobot Interactions.

\section{Different Approaches to the Regulative Problem}

\subsection{Introduction}

Sexual Robots (SRs), today mainly with a female aspect, aims to reproduce as believably as possible a human sexual interaction (Sullins 2012), to satisfy users' sexual desires. These machines will not perform their sexual functions only from a physicalmechanical point of view (Scheutz 2011). In fact, for this type of necessity, countless types of objects are already marketed, thought to increase the physical possibility of enjoying pleasure. On the contrary, SRs won't mimic only the physical aspects of sexual intercourse, but also the relational and emotional ones.

There already are some examples of Sexual Robots on the market, although they are in an early stage (Danaher 2017). These robots can interact physically and verbally with the user, using a predefined set of behaviors (Bendel 2016). One of the most interesting 
technologies today on the market is Roxxxy, a full-size interactive sexual gynoid. Roxxxy has a human-like skin, a good physical resemblance of a real woman and, most important, it interacts verbally adapting her personality to user communicative feedbacks.

The site marketing Roxxxy, True Companion, promise that Roxxxy(s):

"Can hear what you say, speak, feel your touch, move their bodies, are mobile and have emotions and a personality. Additional personalities may be taught to the robot. The additional personality profiles provided with Roxxxy assume very unique sexual characteristics. For example, there is "Wild Wendy" which is very adventurous. There is also "Frigid Farah" that is very reserved and does not always like to engage in intimate activities."

Other companies are producing Sexual Robots with various degrees of human resemblances and autonomy, while the market for these products increases, albeit still limited. Moreover, an online community is born (Su et al. 2019), where users are exchanging experiences and advise on the use of sexual dolls and robots.

\subsection{A Debated Topic}

The starting point of the scholar discussion about Sexual Robotics could be placed in Levy's book "Love and Sex with Robots" (2009) where the author analyses the nature of human-human sexual interactions in comparison with human-robot sexual interactions. His conclusions are enthusiastic about the introduction of Sexual Robots. After that, the interest for Sexual Robotics spread out in the scientific literature. Scholars are mainly divided between critics and supporters. The main concerns are about the possibility to rape SRs (Strikwerda 2015), the commercialization of children SRs (Maras 2017), the rights of SRs (Gunkel 2015), their moral status (Bendel 2016). Currently, there is a growing debate about Sexual Robots in the scientific community.

Most of the discussion focuses on deciding whether robots should have "rights" and which are the acceptable behaviors to perform on them (Richardson 2016a). Two position are the most supported in this discussion: the first argues that robots, since they have a certain degree of intelligence, must be guaranteed certain rights and a moral status (Bendel 2016). Others argue that they should be treated as objects (Levy 2009), because their ability to mimic intelligent behaviors doesn't make them subjects.

More in general, the discussion is divided between enthusiasts and detractors of Sexual Robots. The former argue that rape and violence will decrease (Devlin 2015), sexual satisfaction of users will increase (Levy \& Loebner 2007), it will contribute to the reduction of prostitution (Levy 2012). The latter argue that they will contribute to increase

\footnotetext{
${ }^{1}$ Some deny Roxxxy existence and claim that is a hoax. For the sake of this discussion, we believe that is not a crucial aspect the actual commercialization of such an artefact, but the fact that is an affordable technology for today's state of art.
} 
the violence (Sparrow 2017) and that they will reproduce the existing gender disparities (Cox-George and Bewley 2018).

Most scholars agree that the predictable spread of Sexual Robotics on the market must happen within a regulative framework in order to avoid unacceptable consequences of this technology, as child sex robots (Maras 2017), the worsening of human relational abilities (Turkle et al. 2006), the reproduction and amplification of gender disparities (Cox-George and Bewley 2018).

\subsection{The Exceptionality of Social Robots}

The one of Sexual Robots is only a specific case of the growing awareness about the disruptive impact of Social Robotics in our society, the multiple and probably still unthinkable uses (and misuses) of autonomous and interactive robots.

Both academia and the civil society largely agree that Social Robots won't simply be other objects in our houses because of their degree of autonomy, adaptivity and relationality (Breazeal 2004). Interactions with robots will supposedly strongly resemble the ones with humans and the "social" nature of these robots will, in any case, produce a deep impact on how we experience our intersubjective interactions. This difference between Social Robots (or interactive AIs) and non-interactive technological objects requires a specific theoretical effort (considering the peculiarity of Social Robots) in order to prevent negative consequences and enhance positive outcomes.

Then, a specific theoretical framework for a regulation of Social robots is needed (Sharkey \& Sharkey 2010), since they are a very peculiar type of objects: autonomous, interactive and social-oriented.

This theoretical framework should:

1. Explain if and on what basis Social Robots and interactive AIs differs from "classical" object (ontologically, socially, relationally, morally).

2. Explain what the consequences of this exceptionality are on different levels: moral, legal, social, regulative (etc.).

3. Describe the possible undesirable effects on human sociality and how to prevent them.

Most of the current literature about the consequences of robots' autonomy deals with the problem of moral status, often declined in the terms of "robots rights" (Gunkel 2018).

The problem of moral status can be summarized with the question "should robots be considered as morally relevant, both passively and actively? Should we give moral consideration to actions performed by and on robots?"

Obviously, the question arises because we are today facing massive advances in the field of robotics and AI and more and more machines are able to autonomously act, evaluating the context in where they are and choosing the best thing to do.

In philosophical terms, it is the first time that a technological human-made object is reaching (or at least trying to achieve) a sort of autonomous agency (Broadbent 2017). So, the problem is declined both in the passive and active moral relevance of autonomous machines: what machines (should and should not) do and what we (should and 
should not) do on machines. As said, most part of the current literature on the moral implications of robots and AIs passes through the concept of "rights": many scholars are asking for giving robotics "rights" (Sharkey 2008) in order to institutionalize robots' relevance to morality. Therefore, most of the discussion is about what rights should we grant to robots and on what basis.

Multiple and different answer have been given to these two questions, deeply interconnected between each other. In most of the cases the claim is that we grant rights not only to humans but also to non-human beings as animals and abstract beings as institutions, groups etc. (Gunkel 2018). Hence, rights are differently distributed among different type of recipients, each of them possessing a certain degree of agency. Since autonomous robots have (or can mimic?) a certain degree of agency, then we should grant certain rights (Tavani 2018). Therefore, a spreading literature tries to establish under what conditions robots are worth of moral consideration and what rights must be granted to them. In our opinion, there are two very problematic aspects of thinking the moral problem in terms of rights to robots. First, the hard-to-support ontological assumption that today's robots genuinely have a certain degree of agency. Moreover, it is hard to state on which universal criteria we should rely to grant certain rights to robots with certain ontological properties. We could claim that the qualifying property for rights is self-awareness, or the ability to suffer pain, or intentionality. All of these options make sense (and are broadly supported), still there is no agreement on which is predominant, or the degree of each to possess in order to be qualified. Anyway, without disregarding this valuable approach, we try to ground the regulative framework on another approach that, in our opinion, could produce faster and broader consensus on a theoretical framework for Social Robots regulation

\section{Social-Relational Approach to a Regulation of Social Robotics and Sexual Robotics}

In this paper we propose a different theoretical approach partially based on the "socialrelational approach" proposed by Coeckelbergh (2010).

We believe that the very relevant part of a debate on Social Robotics (and Sexual Robotics as a part of it) should focus on the consequences for human relationality and sociality, when addressing regulative issues. In fact, apart from possessing or not any ontological property, Social Robots surely differs from any other object for their human-likeness and relationality (Sparrow 2016). So, when addressing regulative questions on the introduction of Social Robots in our interactional context, we should focus on the most urgent issue, in our opinion: how will Social Robots shape human relational settings and the human social sphere?

In order to give an answer, it is necessary to state what relational characteristics make Social Robots different from other type of objects, and why we should have a particular normative framework for them.

In fact, the deontological approach provided an explanation on why we should regulate Social Robotics in an exceptional way and why we should not consider them as 
simple objects, namely stating that robots should be guaranteed with rights on the basis of their peculiar ontological properties.

Instead, from a social-relational approach, we claim that Social Robots are a type of object that deeply reconfigure the user's socio-relational setting because of:

1. Their high degree of verbal, nonverbal and even emotional interactivity and their human resemblance (Sharkey 2008), producing interactional patterns very similar to the human-human interactions ones (Kramer et al. 2011).

2. Their unlimited disposability to user needs and desires. In fact, unlike humans, robots never get bored of interacting and are totally at the user disposal (Turkle 2006)

What matters for a social-relational approach is that a certain interactional object will supposedly produce a significant change on human sociality.

The concerns about the effect of relational technologies on human intersubjectivity is common between scholars debating on regulation of SRs, but few attempts to systematizing this approach have been made.

Maras and Shapiro (2017) suggest that child sex robots could contribute to enhance the pedophile phantasies in subjects at risk. Richardson (2016c) states that Sexual Robots contributes on a distorted consideration of humans" bodies as "consumable goods". Turkle (2006) warns that always-at-disposal Social Robots could be preferred to human companions in the future. Even Levy (2009), in one of his papers, is concerned about the possible transfer of degrading behaviors from robots to humans. Nearly every scholar addressing regulatory issues is concerned, at least in part, with the fact that introducing interactive robots in our interactional space could lead to significant changes in our human-human interactions.

Mark Coeckelbergh (2010) proposes, in opposition to theories of object's morality (e.g. deontology) or subject's morality (e.g. virtue ethics) a social-relational theory, which we accept as the ground for our discussion. The main conclusions of his approach are:

- We should care about how robots appear to users, not about the ontological properties.

- We should intend the relation between users and Social Robots as socially (and culturally, historically etc.) contextualized, when dealing with regulative and moral questions.

- If, on one side, the relation between the subject and the interactional object is shaped by the social context, the intersubjective results of this relation will in turn shape the social sphere.

Within Coeckelbergh's social-relational approach, we want to provide a conceptual framework of the reason why (and how, to what extent) interacting with a social robot brings significant changes in human-human interactions.

We will take in consideration the specific case of Sexual Robots, a subset of Social Robots. In fact, we believe that they are an interesting case study to clearly analyze the intersubjective implications of interacting robots. The attempt to extend our conclusion about Sexual Robots to Social Robots in general, will be matter of forthcoming works. In the next section we ground a psychoanalytical framework for Sexual Robot implications in users psychological setting. Afterwards we propose the concept of "Subjective Reference" as a relevant mechanism producing the intersubjective issues of Sexual 
Robots. Then, we suggest our conceptualization of this mechanism as a possible keyelement in order to produce an effective regulative framework for Sexual Robots and Social Robots in general.

\subsection{Sexual Robots and Their Limitless Availability}

The socio-relational approach to sexual robotics allows us to develop important considerations on how the "relationship" with these objects will affect both the people who directly interact with them and the society in general.

First, we contextualize this approach within a psychological theoretical framework.

One of the fundamental concepts of the psychodynamic approach is the conflict between the pleasure principle and the reality one. The first, which characterizes particularly early childhood, implicates that the subject requires unlimited satisfaction of his needs and desires, regardless of the limitations that the outside world inevitably poses. The second, that evolve during the development, allows the subject to tolerate frustration, thus satisfying his own wishes to the extent that it is possible, given the limitations of external world. One of the fundamental consequences of the transition from pleasure principle to that of reality, is the possibility of perceiving and tolerating the other subjects as autonomous (Winnicott 1990), endowed with the same rights as the subject her/himself, despite this inevitably limits the satisfaction of his wills (Nicolò 2003). This means that only the acceptance of the reality principle allows individuals to actually establish a relationship with another subject, taking into account her/his needs and rights, empathizing for her/him, considering her/him as a subject (Baron-Cohen 2011).

Within this concept, the choice to use a sexual robot to satisfy sexual needs makes interesting questions arise. Indeed, we can imply that the choice of using a machine rather than relating to a human, is due to an issue in this area of the personality, since the interactions with a machine requires no compromises with the reality principle. Then, doubts arise on how much sexual robot users have reached the following abilities, typical of the health subject:

- Accepting that the satisfaction of desire is not unlimited and tolerating the resulting frustration.

- $\quad$ Being in relationship with another person, perceived as a subject with rights and needs to be respected.

Conversely, replacing humans with machines allows to:

- Be certain that no limitations on the satisfaction of desire will take place.

- Avoid confrontation with another subject that can advance needs and provide frustration.

This choice can therefore be considered a regressive movement to a psychological setting in which one demands everything and immediately, as in the primary process (Ogden 1989). This mechanism of regression is true for every kind of masturbatory practices, that we define as every practice where only one subject is supposed to have agency and be able to manifest needs and wills.

Moreover, some scholars compared interactions with SRs to ones with sexual workers. As far as prostitution is concerned, it is necessary to underline that despite the sex 
worker is paid, thus can become equal to an object at the phantasmatic level for the user, she always remains a subject. The sex worker does not lose her inalienable rights and she sets specific rules that must be respected. Moreover, the fact that sexual work is paid, imply a negotiation between to subjects. Furthermore, it is in any case a relational act involving a risk of frustration. For example, the subject may fear a criticism of his sexual performance, though not expressed by the sexual worker. Therefore, the relationship with a sex worker remains a relational act, with all the problems that this can cause, although deprived of some characteristics of intersubjective interactions. In the case of Sexual Robots, user faces an interactive object, mimicking a subject, providing unlimited fulfilment of desire.

Therefore, we claim that the use of sexual robots differs from masturbation and prostitution for one prominent reason. In the masturbation, even with the use of particular objects, the subject is actually alone. In interacting with sexual workers the subject is, as we said, still in a real relational act.

The sexual robot stands in a middle way, it is actually a machine, so the user is alone and he is not relating, however a relationship is simulated, not only through the sexual act but also through a series of complementary actions like talking, looking at eachother, approaching in a "human" way (Richardson 2016c).

The sexual robot in fact, does not represent just one part of the human body, such as, for example, a vibrator. Rather it depicts the whole human body and it also tries to simulate the abilities that are proper to human, like linguistic interactions. It implies that if with another masturbatory object only a sexual act is simulated, Sexual Robots reproduce a whole human relationship, not just at its sexual level.

The sexual robot is then an object that is "subjectivized" where user can project a simulated relational satisfaction of his wills and needs (Richardson 2016b). What consequences can this feature have on the user of a sexual robot?

Of course, the differences between a masturbatory act and a relational one fade and this involves multiple consequences. As the object becomes "subjectivized", it is possible that, conversely, human subjects become "objectified". It is therefore possible that a sexual robot user will reproduce, in human-human interactions, some behavior which, if acceptable with a robot, will be unacceptable in a human interaction.

This second implication of human-robot interactions:

1) Directly derives from the nature of human-robot interactions, namely the limitless availability of robots and its "subjectification".

2) Brings many consequences in human-human interactions, since the interactional setting in behaving with robots could be transferred on relations with humans.

3) Deeply impact social and relational sphere of humanity and it is highly relevant for a social-relational regulative framework.

Then, we explain the shift of the relational setting from human-robot interactions to human-human interactions introducing the concept of "subjective reference" 


\subsection{The Concept of "Subjective Reference" and its Relevance for the Regulative Approach}

Sexual Robots producers want them as realistic as possible, to remove any noticeable difference between humans and robots. Today, commercialized SRs reproduce mostly women (Sparrow 2017). They must appear and behave like real humans because users are searching for a substitute of human-human sexual interactions and will supposedly prefer the most similar one (Levy 2009). But, if the robot is chosen largely (or uniquely?) for its similarity to humans, then it is not sexually relevant in itself but in place of something else, namely the thing it tries to mimic and resemble.

In other words, we claim that (except from a minimum part of users fetishizing the very fact that is a machine) users are not enjoying a sexual intercourse with a SR because robots are objects. Users will enjoy it because the object looks like a human subject (objectified and fully under user control).

Therefore, when the user sexually interacts with an SR, his/her sexual phantasies are not focused on the robot itself, but on the human partner which the robot is embodying and mimicking for user's pleasure. The SR operates as a reference (a representant) that enables sexual phantasies (actually directed to a human subject) to be realized on a substitutive object. The human subject is physically absent but is symbolically the real target of all the actions the user will accomplish on SR's body.

In this sense, we claim that a SR is "Subjective Reference": a constant reference to a human subject, a "subjectified" object. We claim that, for the user experience, every action performed upon a Sexual Robot is implicitly directed toward a human subject, including obviously degrading and violent practices.

We believe that this shifting mechanism could severely impact on user sociality and behaviors acceptability. The SRs unlimited availability to user's needs and phantasies could normalize, in the user experience, the expectation of a full and unnegotiated satisfaction of his sexual wills also in human-human interactions (Richardson 2016c).

In addition, we should consider that the most probable users of SRs are subjects with poor or no sexual experiences or subjects with peculiar sexual desires, hard to express in human-human interactions (Cox-George et al. 2018). Most of these unacceptable (or hardly sharable) phantasies in human-human sexual interactions are those violent and degrading. If we allow users - mainly male since SRs are mainly gynoid - to freely express these phantasies on SRs, this could severely modify users' sexual expectations and behaviors acceptability, normalizing aggressive and degrading approaches to sexuality. In fact, since actions performed on SRs are phantasmatically transferred on a human subject, so it will be the presumption of an unlimited availability to any sexual behavior.

Summarizing, we support that:

1) Sexual Robots are sexually relevant only (or for the most part) for their similarity with humans.

2) Every sexual action performed on robots is symbolically referring to a represented human subject; we call this mechanism "subjective reference". 
3) Since robots allow any form of sexual behavior to be performed on them, including violent and degrading, this expectation could (and probably will) be transferred in human-human sexual interactions.

In this paper we do not analyze the specific social and relational implications of Sexual Robotics under the concept of subjective reference. Further work will link this concept with a possible increase of sexism in the social sphere and with a reduction of the social perception of rape severity.

We propose that of "subjective reference" as a conceptual tool useful to:

1) Analyze and forecast possible consequences of Social Robots commercialization

2) Produce design strategies to be implemented in robots to avoid (or reduce) users misuse of Social Robots.

3) Ground a regulative framework of Social Robotics, dealing with the severe implications for sociality and relationality.

In fact, a precise theorization of why and how Sexual Robots - and Social Robots in general - will impact human sociality and relationality allow us to foresee and prevent future negative implications of specific traits of Sexual Robots' behavior.

A regulative framework based (or directly involving) the concept of "subjective reference" takes in strong consideration not the action performed on the robot, valuing its rightness or wrongness in itself. This approach considers primarily if and how this action could impact on human-human relations: we want to assess in which measure the user relational setting will be modified by the HR interaction. The reason why this modification could occur is explained by the concept of Subjective Reference: interactions with robots (and a good example are Sexual Robots) are not meaningful in themselves for the user, but because they enable a shifting mechanism toward a human subject. This means that a regulative framework should limit the unacceptable actions on robots that will strongly refer to a human and, therefore, modify user attitude to relationality in general.

Surely, we find a difficulty in implementing such a theoretical framework: we need a threshold stating a limit to actions performable on robots. This must rely on a precise description of interactional shift between human-robot interactions and human-human ones. We are working on this objective on our next work, in which we will apply the interactionist theory of relationship, derived from Palo Alto school.

Then, we will be able to implement design strategies able to reduce (or even eliminate) the risk of a severe worsening of users' relational abilities and behavior acceptability, by precisely understanding how and why a H-R interactional setting may be transferred to $\mathrm{H}-\mathrm{H}$ interactions.

\section{Conclusions}

In this paper we claimed the greater effectiveness of a social-relational approach over a deontological one. We underlined the limits of deontologies, namely the heavy ontological assumptions that these approaches need. 
We agreed with a social-relational approach that, in our opinion, eases the grounding of a shared theoretical approach in order to produce a regulative framework and forecast Sexual Robots issues. We proposed, under a psychological approach, a conceptualization of the mechanism enabling human-robot interactions to modify human sociality and relationality: the concept of "subjective reference". We supported that the limitless availability of Sexual Robots will result in an identical expectation in the human-human sexual interactions. Finally, we discussed the importance of this conceptualization for future regulative approaches.

In this paper we didn't apply to specific cases the concept of subjective reference, this will be developed in next works. However, we believe the reader may easily guess the possible implications of this conceptualization. For instance, the possibility to rape an interacting robot - able to mimic a sexual appreciation of this violent behavior could have severe consequences for men's respect of women sexual consent. The habit of behave violently may normalize this kind of acts and, slowly, change social norms.

We choose Sexual Robotics to present this issue because it is far more visible than in any other case the risk we are facing with the absence of a regulation. Nevertheless, we believe that the same mechanism occurs in any interactions with Social Robots, given that the machine has good abilities in mimicking humans. With Sexual Robots we believe that is far simpler that the shift occurs because of the peculiarity of sexual impulse.

Next works will deal with specific cases in which the mechanism of subjective reference occurs in order to refine the concept and show its applicability.

The desired goal is to come to a shared theoretical approach useful for a normative and regulative framework on Social Robots.

\section{References}

1. Baron-Cohen, S. (2011) Zero degrees of empathy: A new theory of human cruelty. Penguin UK.

2. Bendel, O. (2016). Sex Robots from the Perspective of Machine Ethics. In International Conference on Love and Sex with Robots (pp. 17-26). Springer

3. Breazeal, C. L. (2004). Designing sociable robots. MIT press

4. Broadbent, E. (2017). Interactions with robots: The truths we reveal about ourselves. Annual review of psychology, 68, 627-652

5. Coeckelbergh, M. (2010). Robot rights? Towards a social-relational justification of moral consideration. Ethics and Information Technology, 12(3), 209-221.

6. Cox-George, C., \& Bewley, S. (2018). I, Sex Robot: the health implications of the sex robot industry. BMJ Sex and Reproductive Health

7. Danaher, J. (2017). Robotic rape and robotic child sexual abuse: should they be criminalized? Criminal law and philosophy, 11(1), 71-95.

8. Devlin, K. (2015). In defence of sex machines: why trying to ban sex robots is wrong. The Conversation.

9. Gunkel, D. J. (2015). The Rights of Machines: Caring for Robotic Care-Givers. In Machine Medical Ethics (pp. 151-166). Springer International Publishing.

10. Gunkel, D. J. (2018). Robot rights. MIT Press. 
11. Gunkel, D. J. (2018). The other question: can and should robots have rights?. Ethics and Information Technology, 20(2), 87-99

12. Levy, D., \& Loebner, H. (2007). Robot prostitutes as alternatives to human sex workers. In IEEE international conference on robotics and automation, Rome (Vol. 14).

13. Levy, D. (2009). Love+ sex with robots: The evolution of human-robot relationships. Duckworth Overlook.

14. Levy, D. (2009). The ethical treatment of artificially conscious robots. International Journal of Social Robotics, 1(3), 209-216

15. Levy, D. (2012). The ethics of robot prostitutes. Robots Ethics: The Ethical and Social Implications of Robotics, op. cit, 223-231.

16. Krämer, N. C., Eimler, S., von der Pütten, A., \& Payr, S. (2011). Theory of companions: what can theoretical models contribute to applications and understanding of human-robot interaction?. Applied Artificial Intelligence, 25(6), 474-502

17. Maras, M. H., \& Shapiro, L. R. (2017) Child Sex Dolls and Robots: More Than Just an Uncanny Valley. Journal of Internet Law

18. Nicolò, A. M. (2003). Appunti a proposito di una teoria dei rapporti soggettuali. Interazioni 2(20), 55-61.

19. Ogden, T. H. (2004) The primitive edge of experience, Karnak, 1989.

20. Richardson, K. (2016a). Sex robot matters: slavery, the prostituted, and the rights of machines. IEEE Technology and Society Magazine, 35(2), 46-53.

21. Richardson, K. (2016b). Technological animism: The uncanny personhood of humanoid machines. Social Analysis, 60(1), 110-128.

22. Richardson, K. (2016c). The asymmetrical 'relationship': parallels between prostitution and the development of sex robots. ACM SIGCAS Computers and Society, 45(3), 290-293.

23. Strikwerda, L. (2015). Present and future instances of virtual rape in light of three categories of legal philosophical theories on rape. Philosophy \& Technology, 28(4), 491-510.

24. Sharkey, N., \& Sharkey, A. (2010). The crying shame of robot nannies: an ethical appraisal. Interaction Studies, 11(2), 161-190.

25. Sharkey, N. (2008). The ethical frontiers of robotics. Science, 322(5909), 1800-1801.

26. Scheutz, M. (2011). 13 The Inherent Dangers of Unidirectional Emotional Bonds between Humans and Social Robots. Robot ethics: The ethical and social implications of robotics, 205.

27. Sparrow, R. (2004). The turing triage test. Ethics and Information Technology, 6(4), 203213.

28. Sparrow, R. (2016). Robots in aged care: a dystopian future?. AI \& society, 31(4), 445-454.

29. Sparrow, R. (2017). Robots, Rape, and Representation. International Journal of Social Robotics, 9(4), 465-477.

30. Su, N. M., Lazar, A., Bardzell, J., \& Bardzell, S. (2019). Of Dolls and Men: Anticipating Sexual Intimacy with Robots. ACM Transactions on Computer-Human Interaction (TOCHI), 26(3), 13.

31. Sullins, J. P. (2012). Robots, love, and sex: the ethics of building a love machine. IEEE transactions on affective computing, 3(4), 398-409.

32. Tavani, H. (2018). Can social robots qualify for moral consideration? Reframing the question about robot rights. Information, 9(4), 73.

33. Turkle, S., Taggart, W., Kidd, C. D., and Dasté, O. (2006). Relational artifacts with children and elders: The complexities of cybercompanionship. Connection Science 18(4): 347-361.

34. Winnicott, D. W. (1990). Creativity and its origins. Essential papers on the psychology of women, 132-145. 